\title{
Rasgos y dimensiones de la personalidad en estudiantes de la Universidad Nacional de Chimborazo
}

\section{Personality traits and dimensions in students of the National University of Chimborazo}

\author{
Autores: \\ Diego Armando Santos Pazos ${ }^{1}$ \\ Luis Gonzalo Erazo Salcedo ${ }^{1}$ \\ Javier Oscar Mogrovejo Gualpa ${ }^{2}$ \\ ${ }^{1}$ Universidad Nacional de Chimborazo, Riobamba-Ecuador. \\ ${ }^{2}$ Universidad de Guayaquil, Guayaquil-Ecuador.
}

Autor de correspondencia: Diego Armando Santos Pazos, Universidad Nacional de Chimborazo, Dirección postal: Ave. Antonio José de Sucre, Km 1 1⁄2, Riobamba, Chimborazo, Ecuador, email: dsantos@unach.edu.ec, teléfono: 0996647577.

\section{RESUMEN}

La investigación tuvo como objetivo, determinar los rasgos y dimensiones de personalidad dominantes en estudiantes de diferentes carreras pertenecientes a la Universidad Nacional Chimborazo (UNACH). Se utilizó una metodología cuantitativa descriptiva, el análisis de datos fue con el paquete de datos estadísticos SPSS versión 23IBM. El instrumento empleado para medir las variables fue el Cuestionario 16PF-5 de Cattell (1993), con el cual se obtuvieron los rasgos y dimensiones globales de personalidad. Para el análisis se prefirió la teoría de los rasgos de la personalidad, incorporando las dimensiones globales: neuroticismo, apertura, afabilidad, extroversión y rectitud. Dentro de los resultados del estudio, se desprende que existen ciertos rasgos dominantes en la muestra como son la inestabilidad emocional y el rasgo de despreocupado. En cuanto a las dimensiones, el principal hallazgo fue la ansiedad alta. Estos tres aspectos de la individualidad que se destacan en el estudio por sus características pueden influir en el desempeño integral de los estudiantes.

Palabras clave: cuestionario de factores de personalidad de Cattell, estudiantes, rendimiento académico.

\begin{abstract}
This research aimed to determine the dominant personality traits and dimensions in students of different schools belonging to the National University Chimborazo (UNACH). A descriptive quantitative methodology was used. The data analysis was carried out using the statistical program SPSS version 23IBM. The Questionnaire 16PF-5 of Cattell (1993) was the instrument applied to measure the variables in order to get the global personality traits and dimensions. The theory of personality traits was preferred for analysis, incorporating the global dimensions:
\end{abstract}


neuroticism, openness, affability, extroversion and rectitude. The results showed that there were certain dominant features in the sample such as: emotional instability and carefree trait. The main finding was high anxiety talking about the dimensions. These three aspects of individuality that are highlighted in the study by their characteristics may influence the overall performance of students.

Keywords: Cattell Personality Factor Questionnaire, Students, Academic Performance.

\section{INTRODUCCIÓN}

Partiendo de la premisa que los rasgos de personalidad representan elementos imprescindibles en el estudio de la conducta, desde su naturaleza dinámica, consistente y duradera; en los contextos educativos universitarios, estos permiten inferir sentimientos, pensamientos y hábitos de los alumnos; los que resultan clave en el desenvolvimiento dentro de la institución y el posterior éxito profesional. ${ }^{(1)}$

La personalidad, vista como categoría psicológica, resulta la expresión de la organización dinámica de los sistemas psicofísicos en el individuo, mediante los cuales se establecen ajustes únicos en su ambiente. ${ }^{(2)}$ Para el autor, la organización dinámica de la personalidad resulta un proceso de desarrollo e integración, sin el cual se puede desencadenar una psicopatología.

Según Suárez Álvarez y Pedrosa, la American Psychological Association (APA) define la personalidad como la estructuración de comportamientos y características del individuo, que posibilitan el proceso de adaptación única a la vida y que incluyen: rasgos, pulsiones, intereses, autoconcepto, valores, capacidades y patrones emocionales. ${ }^{(3)}$

Cattell $^{(4)}$ manifiesta que el estudio de la personalidad permite realizar predicciones acerca del comportamiento, actitud, valores y roles del individuo atendiendo a las características del contexto. La forma en que se manifiestan los rasgos, de conjunto con las experiencias y aprendizaje adquirido a lo largo de la vida, constituye un indicador de la capacidad de los estudiantes para realizar las actividades académicas.

Partiendo de ese principio, el mismo autor ${ }^{(4)}$ estableció un esquema para el análisis factorial a través de un cuestionario que incluye 16 rasgos, que de manera aditiva y lineal estructuran la personalidad en cada individuo.

La personalidad constituye un tema de investigación de interés en el campo de la Psicología. Eysenck $^{(5)}$ manifiesta que es la suma total de los patrones de conducta existentes y potenciales de una persona, los que son determinados por la herencia y el ambiente. Mischel ${ }^{(6)}$ la describe como patrones típicos de conducta, emociones y pensamientos que caracterizan la adaptación del individuo a las situaciones de la vida.

Sin embargo, Allport ${ }^{(7)}$ coincide en establecer al rasgo como la unidad primaria de la personalidad, a los que define como un sistema neuropsíquico focal y generalizado, único en cada indivi- 
duo, el que tiene la capacidad para generar estímulos funcionalmente equivalentes e iniciar y guiar formas consistentes de conductas adaptativas y expresivas.

El análisis factorial propuesto por Cattell, ${ }^{(8)}$ desde un punto de vista empírico, permite englobar y analizar la estructura de la personalidad a partir de las siguientes dimensiones: extroversión, afabilidad, neuroticismo, rectitud y apertura.

La extroversión es una dimensión que expresa dominio y sumisión; la que puede predecir conductas sociales. Los sujetos que la presentan tienden a mantener una vida alegre y apasionante, tienen satisfacción al desenvolverse y mantener control durante las relaciones sociales, son amigables, afectuosos y divertidos; además de que, normalmente mantienen relaciones de pareja sin compromisos permanentes y suelen hacer amigos con rapidez. ${ }^{(9)}$

La afabilidad, también conocida como adaptabilidad social o agradabilidad, indica que esos individuos se llevan bien con otras personas, son amigables, bondadosos y poco hostiles; estos no emplean la autoridad para resolver problemas con otras personas y presentan dificultades para establecer relaciones de pareja. Sin embargo, en 1998, Karson et al. ${ }^{(10)}$ observaron que pueden ser suspicaces, no colaborativos e insensibles.

El neuroticismo, en niveles elevados, se muestra en sujetos con labilidad emocional, inseguridad en sí mismo y preocupación excesiva; por el contrario, aquellos con bajos niveles son felices, satisfechos con su vida, estables y controlan sus emociones. Seis rasgos o facetas se relacionan con la inestabilidad emocional: ansiedad, hostilidad, depresión, autoconciencia, impulsividad y vulnerabilidad. ${ }^{(11)}$

La rectitud es un factor que implica asumir los procesos con seriedad, voluntad de logro, control de impulsos, constancia y autovaloración. Las personas con esa cualidad están más motivadas por alcanzar sus metas; al respecto, Barrios Gaxiola y Frías Armenta ${ }^{(12)}$ refieren que estos tienen esa conducta en todos los ámbitos de su vida social: laboral, escolar, familiar y demás.

Otro factor que conduce al crecimiento personal, la creatividad y la disposición para asimilar nuevas experiencias es la apertura. Por lo general, las personas que lo poseen buscan soluciones inteligentes a los problemas. Este se considera el polo opuesto al convencionalismo, el dogmatismo y el apego a lo tradicional. ${ }^{(13)}$

Teniendo en cuenta los elementos teóricos mencionados, los autores del manuscrito que se presenta realizaron un proceso investigativo con el objetivo de determinar los rasgos y dimensiones de personalidad dominantes en estudiantes de la Universidad Nacional Chimborazo (UNACH).

\section{MATERIAL Y MÉTODOS}

Se desarrolló un estudio con enfoque cuantitativo, de tipo observacional descriptivo y de corte transversal; cuyo propósito fue determinar los rasgos de personalidad dominantes y las dimensiones globales, con una muestra constituida por 111 estudiantes de la Universidad Nacional de 
Chimborazo, matriculados en el octavo semestre de las carreras: Ingeniería Civil e Ingeniería Industrial, Derecho, Economía, Terapia Física y Psicología Clínica.

Los datos fueron obtenidos mediante el cuestionario $16 \mathrm{PF}-5^{(8)}$ y analizados por el software adaptado al español por Nicolás Seisdedos Cubero ${ }^{(14)}$ para identificar los rasgos y dimensiones de la personalidad; a través del cual se midieron 16 rasgos de primer orden, estableciéndose a partir de aquello las 5 dimensiones globales.

En la aplicación del mismo se observó que siete de ellos no estaban presentes en la muestra de estudio los cuales pudieron aparecer de forma positiva o negativa, siendo: pensamiento abstracto, sumiso, práctico, sencillo, tradicionalista, dependiente del grupo, desinhibido.

Tabla 1. Dimensiones globales de personalidad con sus respectivos rasgos

\begin{tabular}{|c|c|}
\hline Dimensiones & Rasgos \\
\hline \multirow{5}{*}{ Extraversión } & Afabilidad. \\
\hline & Animación. \\
\hline & Atrevimiento. \\
\hline & Privacidad. \\
\hline & Autosuficiencia. \\
\hline \multirow{4}{*}{ Ansiedad } & $\begin{array}{l}\text { Estabilidad } \\
\text { emocional }\end{array}$ \\
\hline & Vigilancia \\
\hline & Aprensión \\
\hline & Tensión \\
\hline \multirow{4}{*}{ Dureza } & Afabilidad \\
\hline & Sensibilidad \\
\hline & Abstracción \\
\hline & Apertura al cambio \\
\hline \multirow{3}{*}{ Independencia } & Dominancia \\
\hline & Vigilancia \\
\hline & Apertura al cambio \\
\hline \multirow{4}{*}{ Autocontrol } & Animación \\
\hline & Atención a normas \\
\hline & Abstracción \\
\hline & Compulsividad \\
\hline
\end{tabular}

La base de datos obtenida fue ingresada al paquete estadístico SPSS versión 23, la que se programó para procesarlos y emitir las puntuaciones que permitieron establecer los rasgos y dimensiones de la personalidad. 
Los resultados fueron procesados a través de técnicas estadísticas descriptivas; fundamentalmente mediante el análisis de frecuencias absolutas; estos fueron resumidos en tablas y posteriormente graficados.

Los aspectos éticos que se tomaron en cuenta estuvieron dados por la solicitud de las debidas autorizaciones a los directivos correspondientes, así como al respetar la voluntariedad de los sujetos involucrados en el estudio. Los investigadores respetaron los principios de no maleficencia, justicia, beneficencia y autonomía.

\section{RESULTADOS Y DISCUSIÓN}

La población se encuentra comprendida por los estudiantes de las carreras anteriormente mencionadas, con una muestra seleccionada bajo el criterio del investigador, existiendo 55 hombres y 56 mujeres, con una media etaria de 23,53 años $(\delta \pm 3,70)$, su valor mínimo fue 20 años y el máximo 48 años.

Tomando en cuenta un estudio desarrollado en la UNAM, el cual se denomina perfil de personalidad en estudiantes de Psicología, en donde mediante aplicación del test 16PF se obtiene que los estudiantes de los primeros semestres cuentaban con puntajes elevados de ansiedad, además mencionan que existen características de personalidad inadecuadas que habría que modificar como son: los polos negativos de conservadurismo, autocontrol y estabilidad emocional. ${ }^{(15)} \mathrm{De}$ la misma manera se ha podido evidenciar que los estudiantes de la UNACH presentan altamente ansiedad como dimensión global, y la inestabilidad como rasgo dominante.

Por otro lado, en la Universidad Juárez se realizó un estudio sobre personalidad en estudiantes de reciente ingreso a la Facultad de Medicina la cual se conformaba de 163 estudiantes con una edad de $19.4 \pm 0.95$ años; el 58.3\% fueron del género femenino. En orden decreciente, los valores más altos fueron: I (sensibilidad), F (impetuosidad), Q4 (tensión), Q2 (individualismo) y B (solución de problemas), mientras que los que tuvieron valores menores (media menos desviación estándar), en orden de menor a mayor, fueron: Q1 (rebeldía), N (diplomacia), M (imaginación), O (seguridad), G (responsabilidad) y C (estabilidad emocional). ${ }^{(16)}$ Tomando en cuenta la investigación realizada vale mencionar que los estudiantes de la UNACH presentan valores menores en estabilidad emocional y valores mayores en tensión, lo que sugeriría trabajar en estos aspectos para orientar su accionar estudiantil. 
Tabla 2. Rasgos dominantes de la personalidad

\begin{tabular}{|c|c|c|c|c|c|c|}
\hline Género & Rasgos 1 & No & $\%$ & Rasgos 2 & $\mathbf{N}$ & $\%$ \\
\hline \multirow[t]{7}{*}{ Masculino } & Despreocupado & 6 & 10,9 & Ausencia de rasgos & 16 & 29,1 \\
\hline & Inestabilidad & & & & & \\
\hline & Emocional & 22 & 40,0 & Despreocupado & 13 & 23,6 \\
\hline & Inseguro & 10 & 18,2 & Inseguro & 8 & 14,5 \\
\hline & Racional & 7 & 12,7 & Prudente & 6 & 10,9 \\
\hline & Tensionado & 10 & 18,2 & Tensionado & 12 & 21,8 \\
\hline & Total & 55 & 100,0 & Total & 55 & 100,0 \\
\hline \multirow[t]{9}{*}{ Femenino } & Despreocupado & 7 & 12,5 & Ausencia de rasgos & 8 & 14,3 \\
\hline & Inestabilidad & & & & & \\
\hline & Emocional & 30 & 53,6 & Confiado & 1 & 1,8 \\
\hline & Inseguro & 6 & 10,7 & Despreocupado & 9 & 16,1 \\
\hline & Prudente & 6 & 10,7 & Inseguro & 17 & 30,4 \\
\hline & Racional & 2 & 3,6 & Prudente & 2 & 3,6 \\
\hline & Reservado & 3 & 5,4 & Tensionado & 9 & 16,1 \\
\hline & Tensionado & 2 & 3,6 & Tímido & 10 & 17,9 \\
\hline & Total & 56 & 100,0 & Total & 56 & 100,0 \\
\hline
\end{tabular}

En el contexto estudiado, el rasgo de personalidad que tuvo mayor presencia fue inestabilidad emocional con un porcentaje de $40 \%$ en el género masculino y el 53,6\% en el femenino, sin descartar otros rasgos importantes que aparecen con una frecuencia inferior como despreocupado, tensionado e inseguro.

Esos resultados coinciden con la investigación acerca de los factores de personalidad en estudiantes de Psicología en México, desarrollada por Díaz; ${ }^{(17)}$ en la que, mediante la aplicación del cuestionario 16PF a una muestra de 272 estudiantes, se exploraron los polos de la personalidad. Al respecto, entre los rasgos del negativo destacaron los relativos a: expresividad emocional, inteligencia, impulsividad, emotividad, actitud cognitiva, seguridad de sí mismo y ansiedad.

Tabla 3. Dimensiones globales de la personalidad

\begin{tabular}{llrrr}
\hline Género & \multicolumn{1}{c}{ Dimensión } & Frecuencia & Porcentaje \\
\hline Masculino & Válido & Alta Ansiedad & 40 & 72,7 \\
\cline { 3 - 5 } & Bajo Control Social & 9 & 16,4 \\
\cline { 3 - 5 } & Dependencia & 2 & 3,6 \\
\cline { 3 - 5 } & Mentalidad Dura & 4 & 7,3 \\
\cline { 3 - 5 } & Total & $\mathbf{5 5}$ & $\mathbf{1 0 0 , 0}$ \\
\hline \multirow{2}{*}{ Femenino } & Válido & Alta Ansiedad & 17 & 30,4 \\
\cline { 3 - 5 } & & Bajo Control Social & 26 & 46,4 \\
\cline { 3 - 5 } & Dependencia & 8 & 14,3 \\
\cline { 2 - 4 } & & Mentalidad Dura & 5 & 8,9 \\
\cline { 2 - 4 } & Total & $\mathbf{5 6}$ & $\mathbf{1 0 0 , 0}$ \\
\hline
\end{tabular}


Respecto a las dimensiones de personalidad, se puede evidenciar que la Alta Ansiedad predomina en varones con el 72,7\% y en mujeres el bajo control social con el 46,4\% tomando en cuenta también a la dependencia y la mentalidad dura que aparecen en un rango inferior. Todas las dimensiones globales encontradas en los estudiantes de la Universidad Nacional de Chimborazo clasificadas según el grupo de edad y género (tabla 3).

Una afectación debido a la presencia de ansiedad pudo evidenciarse en otro estudio; en el que los rasgos preponderantes fueron razonamiento, tensión y autosuficiencia; mientras que los menos observados estuvieron dados por la afabilidad y vigilancia. Esos investigadores hallaron diferencias significativas entre ambos sexos en cuanto a estabilidad $6.20 \pm 1.3$ vs.5.17 \pm 1.5 ( $\mathrm{p}=0.018)$ y atrevimiento $6.45 \pm 1.4 \mathrm{vs} .4 .70 \pm 1.4(\mathrm{p}=0.0001){ }^{(18)}$

En cambio, al analizar el perfil de personalidad en deportistas con edades comprendidas entre 14 y 25 años, Marrero, et al. observaron mayores valores de escala en la dimensión "autosuficiencia" (7.25), seguida de "tensión" (6.96). ${ }^{(19)}$

\section{CONCLUSIONES}

El rasgo de personalidad que tuvo mayor presencia fue inestabilidad emocional con independencia del género, mientras que los rasgos menos importantes en el género femenino fueron la racionalidad, la prudencia y la confianza y en el género masculino la despreocupación y la prudencia.

La dimensión de personalidad más afectada en el género masculino fue la alta ansiedad; mientras que en el género femenino: el bajo control social. Las dimensionas menos observadas fueron la dependencia y la mentalidad dura, respectivamente.

Conflictos de interés: los autores declaran que no existen.

Declaración de contribución: Diego Armando Santos Pazos hizo búsqueda de información, análisis y discusión de los resultados, además de desarrollar la redacción del artículo científicoLuis Gonzalo Erazo Salcedo y Javier Oscar Mogrovejo Gualpa realizaron búsqueda bibliográfica y discusión de los resultados, además de participar en la interpretación de los datos y la redacción del artículo.

\section{REFERENCIAS BIBLIOGRÁFICAS}

1. Viteri-Estrella AL, Galarza-Zurita GE. Rasgos de personalidad y estrategias de afrontamiento en los adolescentes estudiantes del bachillerato general unificado de la Unidad Educativa Particular Bautista de la Ciudad de Ambato [Tesis de grado en Internet]. [Ambato]: Universidad Técnica de Ambato; 2016. Disponible en: http://repositorio.uta.edu.ec/bits$\begin{array}{llllllllllllllllllllllllll}\mathrm{t} & \mathrm{r} & \mathrm{e} & \mathrm{a} & \mathrm{m} & / & 1 & 2 & 3 & 4 & 5 & 6 & 7 & 8 & 9 & / & 1 & 9 & 0 & 4 & 5 & / & 2 & / & \mathrm{A} & -\end{array}$ NA\%20VITERI.\%202\%20PROYECTO\%20DE\%20INVESTIGACION.pdf.

2. Torres-Valenzuela A, Velázquez-Hernández GY, Martínez-Luna AA, García-García J, Gómez-Rodríguez M, Jasso-Jiménez LA et al. Rasgos de personalidad en alumnos de reciente 
ingreso a la carrera de medicina. Investigación educ. médica [revista en la Internet]. 2018 Mar [citado 2018 Abr 16]; 7(25): 27-35. Disponible en: http://www.scielo.org.mx/scielo.php?scrip-

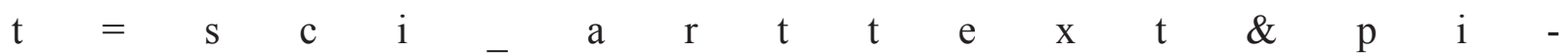
$\mathrm{d}=$ S2007-50572018000100027\&lng=es.http://dx.doi.org/10.1016/j.riem.2017.02.002.

3. Suárez-Álvarez J, Pedrosa I. Evaluación de la personalidad emprendedora: situación actual y líneas de futuro. Papeles del Psicólogo [Internet]. 2016 [citado 2018 Abr 09];37(1): 62-68. Disponible en: https://www.redalyc.org/articulo.oa?id=77844204008.

4. Cattell RB. El análisis científico de la personalidad. Barcelona: Fontanella; 1982.

5. Palacios JR, Martínez R. Descrição das características de personalidade e sócio-culturais dimensões em jovem mexicano. Rev de Psicología [Internet]. 2017 [citado 2018 Abr 19]; 35(2): 453-484. Disponible en: http://www.scielo.org.pe/scielo.php?script=sci_arttext\&pi$\mathrm{d}=\mathrm{S} 0254-92472017000200003 \& \operatorname{lng}=$ es\&nrm $=$ iso.

http://dx.doi.org/10.18800/psico.201702.003.

6. Mischel W. Introduction to personality. New York: Holt, Rinehart and House; 1976.

7. Allport GW. Personality: A psychological interpretation. New York: Henry Holt; 1937.

8. Cattell H, Mead A. The Sixteen Personality Factor Questionnaire 16PF; 2003.

9. Wright T, Reise S. Personaity and unrestricted sexual behavior: Correlations of sociosexuality in Caucasian and Asian college students. Journal of research in Personality; 1997.

10. Karson S. O Dell, WJ. 16 PF Guía de uso clínico. Madrid: Tea ediciones; 1998.

11. Díaz-Paniagua MJ. Relación entre factores de personalidad y la utilización de estrategias de afectividad positiva en estudiantes universitarios [Tesis de grado en Internet]. [Guatemala]: Universidad Rafael Landívar; 2013. Disponible en: http://biblio3.url.edu.gt/Tesario/2013/05/43/Diaz-Maria.pdf.

12. Barrios-Gaxiola MI, Frías-Armenta M. Factores que influyen en el desarrollo y rendimiento escolar de los jóvenes de bachillerato. Rev Colomb de Psicología [Internet]. 2016 [citado 2018 Abr 21]; 25(1): 63-82. Disponible en: https://dialnet.unirioja.es/descarga/articulo/5395094.pdf. doi: $10.15446 /$ rcp.v25n1.46921.

13. Hurtado Bravo ED, Marconi Martínez LI. Perfil de personalidad y liderazgo del personal de mandos medios del área de producción de dos empresas multinacionales con presencia en la ciudad de Cuenca [Tesis de maestría en Internet]. [Guayaquil]: Universidad Espíritu Santo; 2017. Disponible en: http://repositorio.uees.edu.ec/bitstream/123456789/2254/1/V14.\%20DIANA\%20HURTADO\%20TT\%20R.pdf.

14. Campos-Ríos MM. Personalidad y variables clínicas en adolescentes a través del inventario de evaluación de la personalidad para adolescentes (PAI-A) [Tesis Doctoral en Internet]. Málaga: Universidad de Málaga; 2017. [citado 09 de agosto de 2018]. Disponible en: https://riuma.uma.e s/x m lu i/bits trea m/hand le/10630/15669/ T D _ CAMPOS_RIOS_Maria_del_Mar.pdf?sequence=1\&isAllowed=y. 
15. Aragón-Borja LE. Perfil de personalidad de estudiantes universitarios de la carrera de Psicología. El caso de la Facultad de Estudios Superiores Iztacala. Perfiles educativos [Internet]. 2011 [citado 03 de agosto de 2018]; 33(133): 68-87. Disponible en: http://www.scielo.org.mx/scielo.php?script=sci_arttext\&pid=S0185-26982011000300005\&lng=es\&tlng=es.

16. Torres-Valenzuela A, Velázquez-Hernández GY, Martínez-Luna AA, García-García J, Gómez-Rodríguez M, Jasso-Jiménez LA, Ortiz-Valdivia D. Rasgos de personalidad en alumnos de reciente ingreso a la carrera de medicina. Int Ed Med. [Internet]. 2017. [citado 03 de agosto de 2018]; 124: 2-9. Disponible en: https://www.sciencedirect.com/science/article/pii/S2007505717301692.

17. Díaz-Contreras S, Díaz-Reséndiz FJ. Factores de personalidad en estudiantes de psicología en México. Enseñanza e Investigación en Psicología [Internet] 2017; 22(3): 353-363. [citado 18 de octubre de 2018]. Disponible en: http://www.redalyc.org/pdf/292/29255775009.

18. Ruiz-Zavala JH, Enríquez-Sandoval ML, Gómez-Sánchez EA, León-Hernández SR. Relación entre rasgos y tipos de personalidad con la especialidad médica elegida por un grupo de médicos residentes. Investigación educ. médica [Internet]. 2016 Dic [citado 10 de agosto de 2018]; 5(20): 238-243. Disponible en: http://www.scielo.org.mx/scielo.php?script=sci_arttext\&pid=S2007-50572016000400238\&lng=es. http://dx.doi.org/10.1016/j.riem.2016.03.002.

19. Marrero G. Martín-Albo J. y Nuñez J. Perfil de personalidad del tenista. Rev Psicol Dep [internet]. 2000 [citado 14 de octubre de 2018]; 9(12): 21-36. Disponible en: https://www.rpd-online.com/article/view/70/70.

Recibido: 23 de octubre de 2018

Aprobado: 21 de enero de 2019 